\title{
The absolute continuity of the invariant measure of random iterated function systems with overlaps
}

by

\author{
Balázs Bárány (Budapest) and Tomas Persson (Warszawa)
}

\begin{abstract}
We consider iterated function systems on the interval with random perturbation. Let $Y_{\varepsilon}$ be uniformly distributed in $[1-\varepsilon, 1+\varepsilon]$ and let $f_{i} \in C^{1+\alpha}$ be contractions with fixpoints $a_{i}$. We consider the iterated function system $\left\{Y_{\varepsilon} f_{i}+a_{i}\left(1-Y_{\varepsilon}\right)\right\}_{i=1}^{n}$, where each of the maps is chosen with probability $p_{i}$. It is shown that the invariant density is in $L^{2}$ and its $L^{2}$ norm does not grow faster than $1 / \sqrt{\varepsilon}$ as $\varepsilon$ vanishes.

The proof relies on defining a piecewise hyperbolic dynamical system on the cube with an SRB-measure whose projection is the density of the iterated function system.
\end{abstract}

1. Introduction and statements of results. Let $\left\{f_{1}, \ldots, f_{l}\right\}$ be an iterated function system (IFS) on the real line, where the maps are applied according to the probabilities $\left(p_{1}, \ldots, p_{l}\right)$, with the choice of the map random and independent at each step. We assume that for each $i, f_{i}$ maps $[-1,1)$ into itself so that the image is bounded away from -1 and 1 , and $f_{i} \in$ $C^{1+\alpha}([-1,1))$. Let $\nu$ be the invariant measure of our IFS, i.e.

$$
\nu=\sum_{i=1}^{l} p_{i} \nu \circ f_{i}^{-1} .
$$

Let $\mu=\left(p_{1}, \ldots, p_{l}\right)^{\mathbb{N}}$ be a Bernoulli measure on the space $\Sigma=\{1, \ldots, l\}^{\mathbb{N}}$. Let $h(\underline{p})=-\sum_{i=1}^{l} p_{i} \log p_{i}$ be the entropy of the left-shift operator with respect to the Bernoulli measure $\mu$. It was proved in [7], for non-linear, contracting on average, iterated function systems (and later extended in [3]) that

$$
\operatorname{dim}_{\mathrm{H}}(\nu) \leq h /|\chi|,
$$

where $\operatorname{dim}_{H}(\nu)$ is the Hausdorff dimension of the measure $\nu$, and $\chi$ is the Lyapunov exponent of the IFS associated to the Bernoulli measure $\mu$.

2010 Mathematics Subject Classification: Primary 37C40; Secondary 37H15.

Key words and phrases: iterated function system, absolute continuity, random perturbation. 
One can expect that, at least "typically", the measure $\nu$ is absolutely continuous when $h /|\chi|>1$. Essentially the only known approach to this is transversality. For example, for the linear case with uniform contraction ratios, see [8] and [10]. For the linear case and non-uniform contraction ratios, see [5] and [6]. For the non-linear case, see for example [14] and [1]. We note that there is another direction in the study of iterated function systems with overlaps, which is concerned with concrete, but non-typical systems, often of arithmetic nature, for which there is a dimension drop (see for example [4]).

Throughout this paper we are interested in studying absolute continuity with density in $L^{2}$. We will study a modification of the problem, namely we consider a random perturbation of the functions. The linear case was studied by Peres, Simon and Solomyak in [9]. They proved absolute continuity for random linear IFS, with non-uniform contraction ratios and also $L^{2}$ and continuous density in the uniform case. We extend this result by proving $L^{2}$ density with non-uniform contraction ratios and in the non-linear case.

We consider two cases. First let us suppose that for each $i \in\{1, \ldots, l\}$, $f_{i}$ maps $[-1,1)$ into itself, $f_{i}([-1,1))$ is bounded away from -1 and 1 , $f_{i} \in C^{1+\alpha}([-1,1))$ and

$$
0<\lambda_{i, \min } \leq\left|f_{i}^{\prime}(x)\right| \leq \lambda_{i, \max }<1
$$

for every $x \in[-1,1)$. Moreover suppose that for every $i$ the fixed point of $f_{i}$ is $a_{i} \in(-1,1)$, and

$$
i \neq j \Rightarrow a_{i} \neq a_{j} .
$$

Let $Y_{\varepsilon}$ be uniformly distributed on $[1-\varepsilon, 1+\varepsilon]$. Denote the probability measure of $Y_{\varepsilon}$ by $\eta_{\varepsilon}$. Let

$$
f_{i, Y_{\varepsilon}}(x)=Y_{\varepsilon} f_{i}(x)+a_{i}\left(1-Y_{\varepsilon}\right)
$$

for every $i \in\{1, \ldots, l\}$. Then $f_{i, Y_{\varepsilon}}(x)$ is in $[-1,1)$ for all values of $x \in[-1,1)$ and $Y_{\varepsilon}$, provided $\varepsilon$ is sufficiently small. The iterated maps are applied randomly according to the stationary measure $\mu$, with the sequence of independent and identically distributed errors $y_{1}, y_{2}, \ldots$ distributed as $Y_{\varepsilon}$, independent of the choice of the function. The Lyapunov exponent of the IFS is defined by

$$
\chi\left(\mu, \eta_{\varepsilon}\right)=\mathbb{E}\left(\log \left(Y_{\varepsilon} f^{\prime}\right)\right)
$$

and it is easy to see that

$$
\chi\left(\mu, \eta_{\varepsilon}\right)<\sum_{i=1}^{l} p_{i} \log \left((1+\varepsilon) \lambda_{i, \max }\right)<0
$$

for sufficiently small $\varepsilon>0$. Let $Z_{\varepsilon}$ be the random variable

$$
Z_{\varepsilon}:=\lim _{n \rightarrow \infty} f_{i_{1}, y_{1, \varepsilon}} \circ \cdots \circ f_{i_{n}, y_{n, \varepsilon}}(0),
$$


where the numbers $i_{k}$ are i.i.d., with distribution $\mu$ on $\{1, \ldots, l\}$, and $y_{k, \varepsilon}$ are pairwise independent with the distribution of $Y_{\varepsilon}$ and also independent of the choice of $i_{k}$. Let $\nu_{\varepsilon}$ be the distribution of $Z_{\varepsilon}$.

One can easily prove the following theorem.

TheOREM 1.1. The measure $\nu_{\varepsilon}$ converges weakly as $\varepsilon \rightarrow 0$ to the measure $\nu$ satisfying (1.1.

TheOREM 1.2. Let $\nu_{\varepsilon}$ be the distribution of the limit 1.5). Assume that (1.2) and (1.3) hold, and

$$
\sum_{i=1}^{l} p_{i}^{2} \frac{\lambda_{i, \max }}{\lambda_{i, \min }^{2}}<1 .
$$

Then for every sufficiently small $\varepsilon>0, \nu_{\varepsilon}$ is absolutely continuous with respect to Lebesgue measure, with density in $L^{2}$, and there exists a constant $C$ such that the density of $\nu_{\varepsilon}$ satisfies

$$
\left\|\nu_{\varepsilon}\right\|_{2} \leq C / \sqrt{\varepsilon}
$$

REMARK 1. Let

$$
\begin{aligned}
C_{\varepsilon}^{\prime} & =\sqrt{\frac{32}{\left(1-\sum_{i=1}^{l} p_{i}^{2} \frac{(1+\varepsilon) \lambda_{i, \max }}{\left((1-\varepsilon) \lambda_{i, \min }\right)^{2}}\right) C_{\varepsilon}^{\prime \prime}}}, \\
C_{\varepsilon}^{\prime \prime} & =\min _{i \neq j}\left\{\frac{\left|a_{i}-a_{j}\right|+\varepsilon\left(-\left|a_{i}+a_{j}\right|-2\right)}{1-\varepsilon^{2}}\right\} .
\end{aligned}
$$

The proof of Theorem 1.2 will show that $\left\|\nu_{\varepsilon}\right\|_{2} \leq C_{\varepsilon}^{\prime} / \sqrt{\varepsilon}$. Hence we can choose any $C>\lim _{\varepsilon \rightarrow 0} C_{\varepsilon}^{\prime}$.

REMARK 2. Actually the proof of Theorem 1.2 shows that $Z_{\varepsilon}$ conditioned on the perturbations $y_{1, \varepsilon}, y_{2, \varepsilon}, \ldots$ has density in $L^{2}$ for almost all $y_{1, \varepsilon}, y_{2, \varepsilon}, \ldots$

We can state an easy corollary of the theorem.

Corollary 1.3. Let $\left\{\lambda_{i} Y_{\varepsilon} x+a_{i}\left(1-\lambda_{i} Y_{\varepsilon}\right)\right\}_{i=1}^{l}$ be a random iterated function system. Assume that (1.3) holds, and

$$
\sum_{i=1}^{l} \frac{p_{i}^{2}}{\lambda_{i}}<1 .
$$

Then for every sufficiently small $\varepsilon>0, \nu_{\varepsilon}$ is absolutely continuous with respect to Lebesgue measure with density in $L^{2}$, and there exists a constant $C$ such that

$$
\left\|\nu_{\varepsilon}\right\|_{2} \leq C / \sqrt{\varepsilon}
$$

We study another case of random perturbation, namely let $\widetilde{\lambda}_{i, \varepsilon}$ be uniformly distributed on $\left[\lambda_{i}-\varepsilon, \lambda_{i}+\varepsilon\right]$. Let $\left\{\widetilde{\lambda}_{i, \varepsilon} x+a_{i}\left(1-\widetilde{\lambda}_{i, \varepsilon}\right)\right\}_{i=1}^{l}$ be our random 
iterated function system, where $a_{i} \neq a_{j}$ for every $i \neq j$. Let $\underline{\lambda}=\left(\lambda_{1}, \ldots, \lambda_{l}\right)$, and $X_{\underline{\lambda}, \varepsilon}$ be the random variable

$$
X_{\underline{\lambda}, \varepsilon}=\sum_{k=1}^{\infty}\left(a_{i_{k}}\left(1-\widetilde{\lambda}_{i_{k}, \varepsilon}\right)\right) \prod_{j=1}^{k-1} \widetilde{\lambda}_{i_{j}, \varepsilon}
$$

where the numbers $i_{k}$ are i.i.d. with distribution $\mu$ on $\{1, \ldots, l\}$, and $\widetilde{\lambda}_{i_{k}, \varepsilon}$ are pairwise independent. Let $\nu_{\underline{\lambda}, \varepsilon}$ denote the distribution of $X_{\underline{\lambda}, \varepsilon}$. Moreover let $\nu_{\lambda}$ be the invariant measure of the iterated function system $\left\{\lambda_{i} x+\right.$ $\left.a_{i}\left(1-\lambda_{i}\right)\right\}_{i=1}^{l}$ according to $\mu$.

Theorem 1.4. The measure $\nu_{\underline{\lambda}, \varepsilon}$ converges weakly to $\nu_{\underline{\lambda}}$ as $\varepsilon \rightarrow 0$.

To have a statement similar to Theorem 1.2 we need a technical assumption

$$
\min _{i \neq j}\left|\frac{a_{j} \lambda_{i}-a_{i} \lambda_{j}}{\lambda_{i}-\lambda_{j}}\right|>1
$$

TheOREm 1.5. Suppose that (1.9) and (1.3) hold, and moreover

$$
\sum_{i=1}^{l} \frac{p_{i}^{2}}{\lambda_{i}}<1
$$

Then for every sufficiently small $\varepsilon>0$, the measure $\nu_{\underline{\lambda}, \varepsilon}$ is absolutely continuous with respect to Lebesgue measure, with density in $L^{2}$, and there exists a constant $C$ such that

$$
\left\|\nu_{\underline{\lambda}, \varepsilon}\right\|_{2} \leq C / \sqrt{\varepsilon}
$$

REMARK 3. Let

$$
\begin{aligned}
C_{\varepsilon}^{\prime} & =\sqrt{\frac{32}{\left(1-\sum_{i=1}^{l} p_{i}^{2} \frac{\lambda_{i}+\varepsilon}{\left(\lambda_{i}-\varepsilon\right)^{2}}\right) C_{\varepsilon}^{\prime \prime}}}, \\
C_{\varepsilon}^{\prime \prime} & =\sigma \min _{i \neq j} \frac{\left|a_{i} \lambda_{j}-a_{j} \lambda_{i}\right|-\left|\lambda_{i}-\lambda_{j}\right|}{\lambda_{i} \lambda_{j}},
\end{aligned}
$$

where $0<\sigma<1$. As in Remark 1, the proof of Theorem 1.5 will show that $\left\|\nu_{\underline{\lambda}, \varepsilon}\right\|_{2} \leq C_{\varepsilon}^{\prime} / \sqrt{\varepsilon}$ for small $\varepsilon$.

The main difference between Theorem 1.5 and Corollary 1.3 is the random perturbation. Namely, in Theorem 1.5 we choose the contraction ratio uniformly in the $\varepsilon$-neighborhood of $\lambda_{i}$, while in Corollary 1.3 we choose the contraction ratio uniformly in the $\lambda_{i} \varepsilon$-neighborhood of $\lambda_{i}$.

Throughout this paper we will use the method of [11]. 
2. Proof of Theorem 1.2, Let $Q=[-1,1)^{3}$ and $m \in \mathbb{N}$. We partition the cube $Q$ into the rectangles $\left\{Q_{1, k}, \ldots, Q_{l, k}\right\}_{k=0}^{2^{m}-1}$, where

$$
\begin{aligned}
& Q_{i, k}=\{(x, y, z) \in Q:-1+2 \sum_{j=1}^{i-1} p_{j} \leq y<-1+2 \sum_{j=1}^{i} p_{j}, \\
&\left.-1+k 2^{-m+1} \leq z<-1+(k+1) 2^{-m+1}\right\},
\end{aligned}
$$

where we use the convention that an empty sum is 0 . Hence we slice $Q$ into $2^{m}$ slices along the $z$-axis and $l$ slices along the $y$-axis. We thereby get $2^{m} l$ pieces which we call $Q_{i, k}$, according to the definition above.

Let

$$
Q_{i}=\bigcup_{k=0}^{2^{m}} Q_{i, k} .
$$

We define a map $g_{\varepsilon, m}: Q \rightarrow Q$ so that each slice $Q_{i, k}$ is expanded as much as possible in the second and third coordinates. In the first coordinate it is mapped according to a perturbation of $f_{i}$, and hence contracted. Which perturbation is chosen depends on the third coordinate. There is a picture of this in Figure 1.
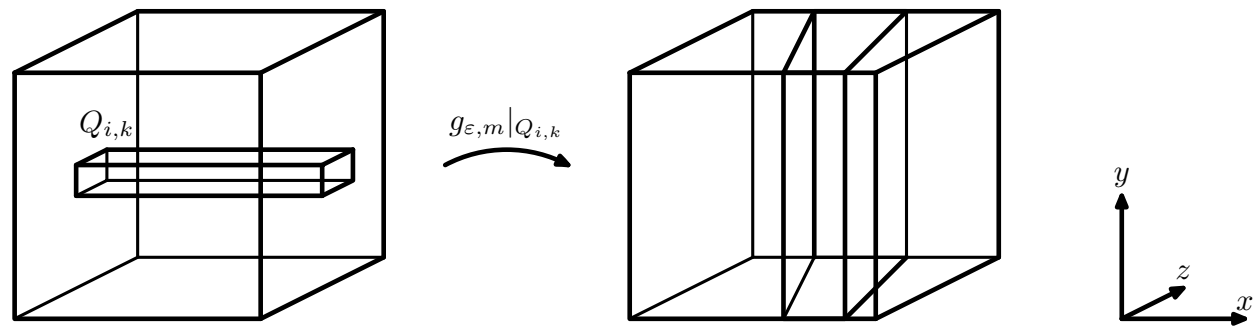

Fig. 1. The action of $g_{\varepsilon, m}$ restricted to $Q_{i, k}$

More precisely, we define $g_{\varepsilon, m}: Q \rightarrow Q$ by setting, for $(x, y, z) \in Q_{i, k}$,

$$
g_{\varepsilon, m}:(x, y, z) \mapsto\left(d(z) f_{i}(x)+a_{i}(1-d(z)), \frac{1}{p_{i}} y+b(y), 2^{m} z+c(z)\right),
$$

where

$$
\begin{array}{ll}
d(z)=1+2^{m} \varepsilon\left(z-\left(-1+(k+1 / 2) 2^{-m+1}\right)\right) & \text { for }(x, y, z) \in Q_{i, k}, \\
b(y)=1-\frac{1}{p_{i}}\left(-1+2 \sum_{j=1}^{i} p_{j}\right) & \text { for }(x, y, z) \in Q_{i, k}, \\
c(z)=2^{m}-2 k-1 & \text { for }(x, y, z) \in Q_{i, k} .
\end{array}
$$

Hence $g_{\varepsilon, m}$ maps each of the pieces $Q_{i, j}$ so that it is contracted in the $x$-direction and fully expanded in the $y$ - and $z$-directions. 
Let $\mathcal{L}_{3}$ be the normalised Lebesgue measure on $Q$. The measures

$$
\gamma_{\varepsilon, m, n}=\frac{1}{n} \sum_{k=0}^{n-1} \mathcal{L}_{3} \circ g_{\varepsilon, m}^{-k}
$$

converge weakly to an SRB-measure $\gamma_{\varepsilon, m}$ as $n \rightarrow \infty$ (see [12] and [13]). The measure $\gamma_{\varepsilon, m}$ is ergodic by the Hopf argument, since $g_{\varepsilon, m}$ is hyperbolic and the stable and unstable manifolds are parallel to the coordinate axes and have maximal extension in the box $Q$. Moreover, let $\nu_{\varepsilon, m}$ be the projection of $\gamma_{\varepsilon, m}$ onto the first coordinate. More precisely, if $E \subset[-1,1)$ is a measurable set, then we define $\nu_{\varepsilon, m}(E)=\gamma_{\varepsilon, m}(E \times[-1,1) \times[-1,1))$.

The measure $\nu_{\varepsilon, m}$ is the distribution of the limit

$$
\lim _{n \rightarrow \infty} f_{i_{1}, y_{1, \varepsilon}} \circ \cdots \circ f_{i_{n}, y_{n, \varepsilon}}(0),
$$

where $y_{i, \varepsilon}$ are uniformly distributed on $[1-\varepsilon, 1+\varepsilon]$, but not independent. However, one can easily prove the following lemma.

Lemma 2.1. The measure $\nu_{\varepsilon, m}$ converges weakly to $\nu_{\varepsilon}$ as $m \rightarrow \infty$.

Let

$$
A_{i}=\left\{(i, 0),(i, 1), \ldots,\left(i, 2^{m}-1\right)\right\} \quad \text { and } \quad A=\bigcup_{i=1}^{l} A_{i} .
$$

If $a=(i, k) \in A$ we will write $\hat{Q}_{a}$ for $Q_{i, k}$. With this notation we have

$$
Q=\bigcup_{a \in A} \hat{Q}_{a} \quad \text { and } \quad Q_{i}=\bigcup_{a \in A_{i}} \hat{Q}_{a}, \quad i=0,1, \ldots, l .
$$

Let $\Theta_{0}=A^{\mathbb{N} \cup\{0\}}$. If $p \in Q$ then there is a unique sequence $\rho_{0}(p)=$ $\left\{\rho_{0}(p)_{k}\right\}_{k=0}^{\infty} \in \Theta_{0}$ such that

$$
g_{\varepsilon, m}^{k}(p) \in Q_{\rho_{0}(p)_{k}}, \quad k=0,1, \ldots
$$

The map $\rho_{0}: Q \rightarrow \Theta_{0}$ is not injective. We have $\rho_{0}(x, y, z)=\rho_{0}\left(x^{\prime}, y^{\prime}, z^{\prime}\right)$ if $y=y^{\prime}$ and $z=z^{\prime}$, but $\rho_{0}(x, y, z) \neq \rho_{0}\left(x^{\prime}, y^{\prime}, z^{\prime}\right)$ otherwise. Hence we can (and will) use the notation $\rho_{0}(y, z)$ instead of $\rho_{0}(x, y, z)$.

We will denote elements in $\Theta_{0}$ by $\boldsymbol{a}, \boldsymbol{b}$ and so on. We let $\sigma$ denote the left shift on $\Theta_{0}$, defined in the usual way.

We can transfer the measures $\gamma_{\varepsilon, m}$ to a measure $\gamma_{\Theta_{0}}$ by $\gamma_{\Theta_{0}}=\gamma_{\varepsilon, m} \circ \rho_{0}^{-1}$.

We let $\Theta$ denote the natural extension of $\Theta_{0}$. That is, $\Theta$ is the set of all two-sided infinite sequences such that any one-sided infinite subsequence of a sequence in $\Theta$ is a sequence in $\Theta_{0}$. The measure $\gamma_{\Theta_{0}}$ defines an ergodic measure $\gamma_{\Theta}$ on $\Theta$ in a natural way. If $\xi: \Theta \rightarrow \Theta_{0}$ is defined by $\xi\left(\left\{i_{k}\right\}_{k \in \mathbb{Z}}\right)=\left\{i_{k}\right\}_{k \in \mathbb{N} \cup\{0\}}$, then we define $\gamma_{\Theta}\left(\xi^{-1} E\right)=\gamma_{\Theta_{0}}(E)$. We can define a map $\rho^{-1}: \Theta \rightarrow Q$ such that $\rho^{-1}(\sigma(\boldsymbol{a}))=g_{\varepsilon, m}\left(\rho^{-1}(\boldsymbol{a})\right)$ for any sequence $\boldsymbol{a} \in \Theta$. 
We note that the $L^{2}$ norm of the density $\nu_{\varepsilon, m}$ is not larger than twice that of the density of $\gamma_{\varepsilon, m}$. If $h_{\nu_{\varepsilon, m}}(x)$ and $h_{\gamma_{\varepsilon, m}}(x, y, z)$ denote the densities of $\nu_{\varepsilon, m}$ and $\gamma_{\varepsilon, m}$ respectively, then by Cauchy-Schwarz's inequality

$$
\begin{aligned}
\left\|\nu_{\varepsilon, m}\right\|_{2}^{2} & \leq \int_{-1}^{1} h_{\nu_{\varepsilon, m}}(x)^{2} d x=32 \int_{-1}^{1}\left(\int_{-1}^{1} \int_{-1}^{1} h_{\gamma_{\varepsilon, m}}(x, y, z) \frac{d y}{2} \frac{d z}{2}\right)^{2} \frac{d x}{2} \\
& \leq 32 \int_{-1}^{1} \int_{-1}^{1} \int_{-1}^{1} h_{\gamma_{\varepsilon, m}}(x, y, z)^{2} \frac{d y}{2} \frac{d z}{2} \frac{d x}{2}=4\left\|\gamma_{\varepsilon, m}\right\|_{2}^{2} .
\end{aligned}
$$

This proves that if $\gamma_{\varepsilon, m}$ has $L^{2}$ density, then so has $\nu_{\varepsilon, m}$, and

$$
\left\|\nu_{\varepsilon, m}\right\|_{2} \leq 2\left\|\gamma_{\varepsilon, m}\right\|_{2} \text {. }
$$

If $p$ is a point in $Q$, then we let $T_{p} Q$ denote the tangent space at $p$. For each $p$ in $Q$ we define the following cone in the tangent space $T_{p} Q$ :

$$
C_{p}=\left\{(u, v, w) \in T_{p} Q:\left|\frac{u}{w}\right|,\left|\frac{v}{w}\right|<\frac{2^{m+1} \varepsilon}{2^{m}-\lambda_{\max , \max }(1+\varepsilon)}\right\},
$$

where $\lambda_{\max , \max }=\max _{i} \lambda_{i, \max }=\max _{i} \sup _{x \in[-1,1)}\left|f_{i}^{\prime}(x)\right|$. The following lemma states that the set of cones $C_{p}$ defines a family of unstable cones, and that images of certain curves intersect transversally. There is an illustration of the transversality in Figure 2 .
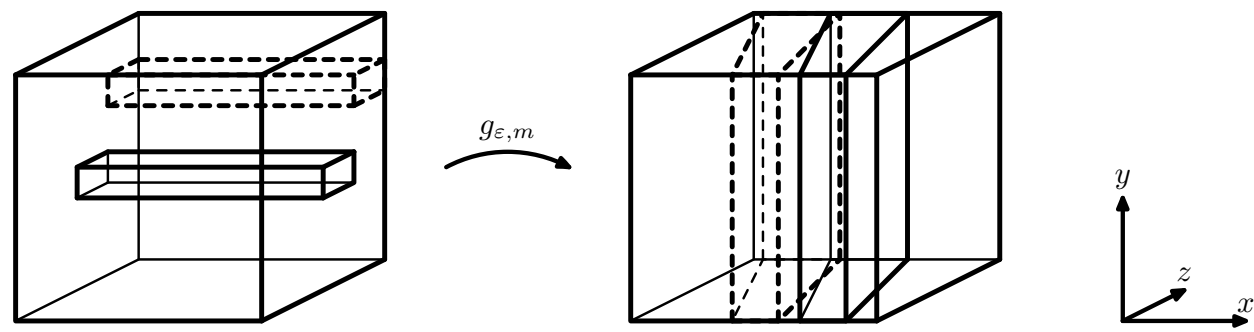

Fig. 2. Any two different $Q_{i, k}$ and $Q_{j, l}$ on the same height $(i=j)$ share the same image, but in the case when $i \neq j$ their images have transversal intersection if they intersect.

Lemma 2.2. The cones $C_{p}$ make up a family of unstable cones, that is, $d_{p} g_{\varepsilon, m}\left(C_{p}\right) \subset C_{g_{\varepsilon, m}(p)}$.

Moreover, for sufficiently large $m$ and every $0<\varepsilon<\min _{i \neq j} \frac{\left|a_{i}-a_{j}\right|}{2+\left|a_{i}+a_{j}\right|}$, if $\zeta_{1} \subset Q_{\xi_{1}}$ and $\zeta_{2} \subset Q_{\xi_{2}}$ are two curve segments with tangents in $C_{p}$ such that $\xi_{1} \in A_{i}$ and $\xi_{2} \in A_{j}, i \neq j$, then if $g_{\varepsilon, m}\left(\zeta_{1}\right)$ and $g_{\varepsilon, m}\left(\zeta_{2}\right)$ intersect, and if $\left(u_{1}, v_{1}, 1\right)$ and $\left(u_{2}, v_{2}, 1\right)$ are tangents to $g_{\varepsilon, m}\left(\zeta_{1}\right)$ and $g_{\varepsilon, m}\left(\zeta_{2}\right)$ respectively, it follows that $\left|u_{1}-u_{2}\right|>C_{\varepsilon, m} \varepsilon$, where

$$
C_{\varepsilon, m}=\min _{i \neq j}\left\{\frac{\left|a_{i}-a_{j}\right|+\varepsilon\left(-\left|a_{i}+a_{j}\right|-2\right)}{1-\varepsilon^{2}}-\frac{4(1+\varepsilon) \lambda_{\max , \max }}{2^{m}-\lambda_{\max , \max }(1+\varepsilon)}\right\} .
$$


Proof. The Jacobian of $g_{\varepsilon, m}$ is

$$
d_{p} g_{\varepsilon, m}=\left(\begin{array}{ccc}
d(z) f_{i}^{\prime}(x) & 0 & 2^{m} \varepsilon\left(f_{i}(x)-a_{i}\right) \\
0 & 1 / p_{i} & 0 \\
0 & 0 & 2^{m}
\end{array}\right)
$$

where $p=(x, y, z) \in Q_{i, k}$. If $(u, v, w) \in C_{p}$, then

$$
d_{p} g_{\varepsilon, m}(u, v, w)=\left(\begin{array}{c}
d(z) f_{i}^{\prime}(x) u+2^{m} \varepsilon\left(f_{i}(x)-a_{i}\right) w \\
\left(1 / p_{i}\right) v \\
2^{m} w
\end{array}\right)
$$

We just need to check that this vector is in $C_{p}$, provided that $m$ is large. This is easily checked, using that $|d(z)| \leq 1+\varepsilon,\left|f_{i}^{\prime}(x)\right| \leq \lambda_{i, \max }$ and $\left|f_{i}(x)-a_{i}\right| \leq 2$. Indeed,

$$
\begin{aligned}
\frac{\left|d(z) f_{i}^{\prime}(x) u+2^{m} \varepsilon\left(f_{i}(x)-a_{i}\right) w\right|}{\left|2^{m} w\right|} \leq \frac{(1+\varepsilon) \lambda_{i, \max }}{2^{m}} \frac{|u|}{|w|}+2 \varepsilon \\
\leq \frac{(1+\varepsilon) \lambda_{i, \max }}{2^{m}} \frac{2^{m+1} \varepsilon}{2^{m}-(1+\varepsilon) \lambda_{\max , \max }}+2 \varepsilon \leq \frac{2^{m+1} \varepsilon}{2^{m}-(1+\varepsilon) \lambda_{\max , \max }}
\end{aligned}
$$

and

$$
\frac{\left|\left(1 / p_{i}\right) v\right|}{\left|2^{m} w\right|} \leq \frac{1}{p_{i} 2^{m}} \frac{2^{m+1} \varepsilon}{2^{m}-(1+\varepsilon) \lambda_{\max , \max }} \leq \frac{2^{m+1} \varepsilon}{2^{m}-(1+\varepsilon) \lambda_{\max , \max }}
$$

proves that $d_{p} g_{\varepsilon, m}\left(C_{p}\right) \subset C_{g_{\varepsilon, m}(p)}$ if $m$ is sufficiently large, so that $2^{m}-$ $(1+\varepsilon) \lambda_{\max , \max }>0$ and $p_{i} 2^{m}>1$.

To prove the other statement of the lemma, assume that $p=\left(x_{p}, y_{p}, z_{p}\right)$ $\in Q_{i}$ and $q=\left(x_{q}, y_{q}, z_{q}\right) \in Q_{j}, i \neq j$, are such that $g_{\varepsilon, m}(p)=g_{\varepsilon, m}(q)=$ $(x, y, z)$. Then, if $p \in Q_{i}$,

$$
d_{p} g_{\varepsilon, m}:(u, v, 1) \mapsto 2^{m}\left(\frac{d\left(z_{p}\right) f_{i}^{\prime}\left(x_{p}\right)}{2^{m}} u+\left(f_{i}\left(x_{p}\right)-a_{i}\right) \varepsilon, \frac{v}{p_{i}}, 1\right) .
$$

Then

$$
f_{i}\left(x_{p}\right)=\frac{x-a_{i}\left(1-d\left(z_{p}\right)\right)}{d\left(z_{p}\right)} \quad \text { and } \quad f_{j}\left(x_{q}\right)=\frac{x-a_{j}\left(1-d\left(z_{q}\right)\right)}{d\left(z_{q}\right)} .
$$

Without loss of generality, assume that $a_{i}>a_{j}$. For simplicity we study the case $x \geq a_{i}>a_{j}$. The proofs for $a_{i} \geq x \geq a_{j}$ and $a_{i}>a_{j} \geq x$ are similar. Then

$$
d_{p} g_{\varepsilon, m}\left(C_{p}\right) \subset\left\{w(u, v, 1): \frac{x-a_{i}}{1+\varepsilon} \varepsilon-\Delta_{i} \varepsilon \leq u \leq \frac{x-a_{i}}{1-\varepsilon} \varepsilon+\Delta_{i} \varepsilon\right\},
$$


where $\Delta_{i}=\frac{2(1+\varepsilon) \lambda_{i, \max }}{2^{m}-\lambda_{\max , \max }(1+\varepsilon)}$. Therefore

$$
\begin{aligned}
\left|u_{2}-u_{1}\right| & \geq \frac{x-a_{j}}{1+\varepsilon} \varepsilon-\frac{x-a_{i}}{1-\varepsilon} \varepsilon-\left(\Delta_{i}+\Delta_{j}\right) \varepsilon \\
& \geq\left(\frac{a_{i}-a_{j}+\varepsilon\left(a_{i}+a_{j}-2\right)}{1-\varepsilon^{2}}-2 \max _{i} \Delta_{i}\right) \varepsilon
\end{aligned}
$$

for every $x \geq a_{i}>a_{j}$. Let $\Delta_{\max }=\max _{i} \Delta_{i}$. Since $0<\varepsilon<\min _{i \neq j} \frac{\left|a_{i}-a_{j}\right|}{2+\left|a_{i}+a_{j}\right|}$, we have

$$
\frac{a_{i}-a_{j}+\varepsilon\left(a_{i}+a_{j}-2\right)}{1-\varepsilon^{2}}>0 .
$$

Therefore

$$
\frac{a_{i}-a_{j}+\varepsilon\left(a_{i}+a_{j}-2\right)}{1-\varepsilon^{2}}-2 \Delta_{\max }>0,
$$

for sufficiently large $m$. By similar methods we have for $a_{i} \geq x \geq a_{j}$,

$$
\left|u_{2}-u_{1}\right| \geq\left(\frac{a_{i}-a_{j}}{1+\varepsilon}-2 \Delta_{\max }\right) \varepsilon
$$

and for $a_{i}>a_{j} \geq x$,

$$
\left|u_{2}-u_{1}\right| \geq\left(\frac{a_{i}-a_{j}-\varepsilon\left(a_{i}+a_{j}+2\right)}{1-\varepsilon^{2}}-2 \Delta_{\max }\right) \varepsilon .
$$

Therefore we can choose

$$
C_{\varepsilon, m}=\min _{i \neq j}\left\{\frac{\left|a_{i}-a_{j}\right|+\varepsilon\left(-\left|a_{i}+a_{j}\right|-2\right)}{1-\varepsilon^{2}}-2 \Delta_{\max }\right\} .
$$

The rest of the section follows Tsujii's article [15].

Proof of Theorem 1.2. For any $r>0$ we define the bilinear form $(\cdot, \cdot)_{r}$ of signed measures on $\mathbb{R}$ by

$$
\left(\rho_{1}, \rho_{2}\right)_{r}=\int_{\mathbb{R}} \rho_{1}\left(B_{r}(x)\right) \rho_{2}\left(B_{r}(x)\right) d x
$$

where $B_{r}(x)=[x-r, x+r]$. It is easy to see that if

$$
\liminf _{r \rightarrow 0} \frac{1}{r^{2}}(\rho, \rho)_{r}<\infty
$$

then the measure $\rho$ has density in $L^{2}$ (see [15]). Moreover

$$
\|\rho\|_{2}^{2} \leq \liminf _{r \rightarrow 0} \frac{1}{r^{2}}(\rho, \rho)_{r} .
$$

Let $\gamma_{z}$ denote the conditional measure of $\gamma_{\varepsilon, m}$ on the set $R_{z}=\{(u, v, w) \in$ $Q: v=y, w=z\}$. Since the one-dimensional Lebesgue measure is invariant under the action of $g_{\varepsilon, m}$ projected to the second coordinate, we conclude 
that $\gamma_{z}$ is independent of $y$ almost everywhere. It follows that

$$
\left\|\gamma_{\varepsilon, m}\right\|_{2}^{2}=\int_{-1}^{1}\left\|\gamma_{z}\right\|_{2}^{2} d z
$$

Let

$$
J(r):=\frac{1}{r^{2}} \int_{-1}^{1}\left(\gamma_{z}, \gamma_{z}\right)_{r} d z .
$$

By the invariance of $\gamma_{\varepsilon, m}$ it follows that

$$
\gamma_{z}=2^{-m} \sum_{i=1}^{l} p_{i} \sum_{a \in A_{i}} \gamma_{g_{\varepsilon, m}^{-a}(z)} \circ g_{\varepsilon, m}^{-a},
$$

where $g_{\varepsilon, m}^{-a}$ denotes the inverse branch of $g_{\varepsilon, m}$ with image in $\hat{Q}_{a}$. Recall that $a \in A_{i}$ means that $a=(i, k)$ for some $k$, so that $\hat{Q}_{a}=Q_{i, k}$ for some $k$. We denote the measure $\gamma_{g_{\varepsilon, m}^{-a}(z)} \circ g_{\varepsilon, m}^{-a}$ by $\sigma_{a, z}$. Then by $(2.3)$ and the definition of $J(r)$,

$$
J(r)=\frac{1}{2^{2 m} r^{2}} \sum_{i=1}^{l} \sum_{j=1}^{l} p_{i} p_{j} \sum_{a \in A_{i}} \sum_{b \in A_{j}} \int_{-1}^{1}\left(\sigma_{a, z}, \sigma_{b, z}\right)_{r} d z .
$$

For fixed $a, b \in A_{i}$,

$$
\begin{aligned}
& \left(\sigma_{a, z}, \sigma_{b, z}\right)_{r} \leq\left(\sigma_{a, z}, \sigma_{a, z}\right)_{r}^{1 / 2}\left(\sigma_{b, z}, \sigma_{b, z}\right)_{r}^{1 / 2} \\
\leq & (1+\varepsilon) \lambda_{i, \max }\left(\gamma_{g_{\varepsilon, m}^{-a}(z)}, \gamma_{g_{\varepsilon, m}^{-a}(z)}\right) \frac{{ }^{1 / 2}}{(1-\varepsilon) \lambda_{i, \min }} \times\left(\gamma_{g_{\varepsilon, m}^{-b}(z)}, \gamma_{g_{\varepsilon, m}^{-b}(z)}\right) \frac{{ }^{1 / 2}}{(1-\varepsilon) \lambda_{i, \min }} \\
\leq & (1+\varepsilon) \lambda_{i, \max } \frac{\left(\gamma_{g_{\varepsilon, m}^{-a}(z)}, \gamma_{g_{\varepsilon, m}^{-a}(z)}\right) \frac{r}{(1-\varepsilon) \lambda_{i, \min }}+\left(\gamma_{g_{\varepsilon, m}^{-b}(z)}, \gamma_{g_{\varepsilon, m}^{-b}(z)}\right) \frac{r}{(1-\varepsilon) \lambda_{i, \min }}}{2} .
\end{aligned}
$$

Moreover, if $a \in A_{i}$ and $b \in A_{j}, i \neq j$, then

$$
\begin{aligned}
&\left(\sigma_{a, z}, \sigma_{b, z}\right)_{r}=\int \sigma_{a, z}\left(B_{r}(x)\right) \sigma_{b, z}\left(B_{r}(x)\right) d x \\
&=\iiint \mathbb{I}_{\{s:|s-x|<r\}}(s) \mathbb{I}_{\{t:|t-x|<r\}}(t) d \sigma_{a, z}(s) d \sigma_{b, z}(t) d x \\
& \leq \iint 2 r \mathbb{I}_{\{(s, t):|s-t|<2 r\}}(s, t) d \sigma_{a, z}(s) d \sigma_{b, z}(t) \\
&=\iint \mathbb{I}_{\left\{(\boldsymbol{c}, \boldsymbol{d}):\left|\rho^{-1}\left(\cdots c_{-2} c_{-1} a \rho_{0}(z)\right)-\rho^{-1}\left(\cdots d_{-2} d_{-1} b \rho_{0}(z)\right)\right|<2 r\right\}}(\boldsymbol{c}, \boldsymbol{d}) \\
& d \gamma_{\Theta}(\boldsymbol{c}) d \gamma_{\Theta}(\boldsymbol{d}) .
\end{aligned}
$$

Let us comment on the notation $\rho_{0}(z)$. Actually $\rho_{0}(z)$ is not defined, but rather $\rho_{0}(x, y, z)$. Recall that $\rho_{0}(x, y, z)$ is independent of $x$ and that we therefore have introduced the notation $\rho_{0}(y, z)$. Moreover, as noticed above, the measures $\gamma_{z}$, and therefore also $\sigma_{a, z}$, are independent of $y$. Hence we can choose arbitrary $x, y$ and let $\rho_{0}(z)$ denote $\rho_{0}(x, y, z)=\rho_{0}(y, z)$. Since all 
the estimates below will be independent of this choice of $y$, we will use the notation $\rho_{0}(z)$ instead of $\rho_{0}(x, y, z)$.

By a change of order of integration we get

$$
\begin{aligned}
\int_{-1}^{1}\left(\sigma_{a, z}, \sigma_{b, z}\right)_{r} d z \leq & 2 r \iint \mathcal{L}_{1}\left(\left\{z: \mid \rho^{-1}\left(\cdots c_{-2} c_{-1} a \rho_{0}(z)\right)\right.\right. \\
& \left.\left.-\rho^{-1}\left(\cdots d_{-2} d_{-1} b \rho_{0}(z)\right) \mid<2 r\right\}\right) d \gamma_{\Theta}(\boldsymbol{c}) d \gamma_{\Theta}(\boldsymbol{d}) .
\end{aligned}
$$

We will now apply Lemma 2.2 to 2.6$)$. Note that

$$
z \mapsto \rho^{-1}\left(\cdots c_{-2} c_{-1} a \rho_{0}(z)\right), \quad \text { and } \quad z \mapsto \rho^{-1}\left(\cdots d_{-2} d_{-1} b \rho_{0}(z)\right)
$$

are two curves with tangents in the cones $C_{p}$. Lemma 2.2 states that these curves have a transversal intersection, if they intersect, so that $\mathcal{L}_{1}\left(\left\{z:\left|\rho^{-1}\left(\cdots c_{-2} c_{-1} a \rho_{0}(z)\right)-\rho^{-1}\left(\cdots d_{-2} d_{-1} b \rho_{0}(z)\right)\right|<2 r\right\}\right) \leq 4 r / C_{\varepsilon, m}$. Hence

By using (2.4) we have

$$
\int_{-1}^{1}\left(\sigma_{a, z}, \sigma_{b, z}\right)_{r} d z \leq \frac{8 r^{2}}{C_{\varepsilon, m} \varepsilon} .
$$

$$
\begin{aligned}
J(r)= & \frac{1}{2^{2 m} r^{2}} \sum_{i=1}^{l} p_{i}^{2} \sum_{a, b \in A_{i}} \int_{-1}^{1}\left(\sigma_{a, z}, \sigma_{b, z}\right)_{r} d z \\
& +\frac{1}{2^{2 m} r^{2}} \sum_{i \neq j} p_{i} p_{j} \sum_{a \in A_{i}} \sum_{b \in A_{j}} \int_{-1}^{1}\left(\sigma_{a, z}, \sigma_{b, z}\right)_{r} d z .
\end{aligned}
$$

We first give an upper bound for the first summand in (2.8), using (2.5) and an integral transformation. By (2.5) we have

$$
\begin{aligned}
& \sum_{a, b \in A_{i}-1} \int_{-1}^{1}\left(\sigma_{a, z}, \sigma_{b, z}\right)_{r} d z \\
& \quad \leq(1+\varepsilon) \lambda_{i, \max } 2^{m} \sum_{a \in A_{i}-1} \int_{-1}^{1}\left(\gamma_{g_{\varepsilon, m}^{-a}(z)}, \gamma_{g_{\varepsilon, m}^{-a}(z)}\right) \frac{r}{(1-\varepsilon) \lambda_{i, \min }} d z \\
& \quad=(1+\varepsilon) \lambda_{i, \max } 2^{m} \sum_{k=0}^{2^{m}-1} 2^{m} \int_{-1+k 2^{-m+1}}^{-1+(k+1) 2^{-m+1}}\left(\gamma_{z}, \gamma_{z}\right) \frac{r}{(1-\varepsilon) \lambda_{i, \min }} d z
\end{aligned}
$$

Hence

$$
\begin{aligned}
& \text { 9) } \frac{1}{2^{2 m} r^{2}} \sum_{i=1}^{l} p_{i}^{2} \sum_{a, b \in A_{i}} \int_{-1}^{1}\left(\sigma_{a, z}, \sigma_{b, z}\right)_{r} d z \\
& \leq \frac{1}{2^{2 m} r^{2}} \sum_{i=1}^{l} p_{i}^{2}(1+\varepsilon) \lambda_{i, \max } 2^{m} \sum_{k=0}^{2^{m}-1} 2^{m} \int_{-1+k 2^{-m+1}}^{-1+(k+1) 2^{-m+1}}\left(\gamma_{z}, \gamma_{z}\right)_{\frac{r}{(1-\varepsilon) \lambda_{i, \min }}} d z
\end{aligned}
$$




$$
\begin{aligned}
& \leq \sum_{i=1}^{l} p_{i}^{2} \frac{(1+\varepsilon) \lambda_{i, \max }}{\left((1-\varepsilon) \lambda_{i, \min }\right)^{2}} \frac{1}{\left(\frac{r}{(1-\varepsilon) \lambda_{i, \min }}\right)^{2}} \int_{-1}^{1}\left(\gamma_{z}, \gamma_{z}\right) \frac{r}{(1-\varepsilon) \lambda_{i, \min }} d z \\
& \leq \max _{i} J\left(\frac{r}{\lambda_{i, \min }(1-\varepsilon)}\right) \sum_{i=1}^{l} p_{i}^{2} \frac{(1+\varepsilon) \lambda_{i, \max }}{\left((1-\varepsilon) \lambda_{i, \min }\right)^{2}} .
\end{aligned}
$$

For the second summand in 2.8 , we use 2.7 to prove that it is bounded by

$$
\begin{aligned}
\frac{1}{2^{2 m} r^{2}} \sum_{i \neq j} p_{i} p_{j} \sum_{a \in A_{i}} \sum_{b \in A_{j}} \int_{-1}^{1}\left(\sigma_{a, z}, \sigma_{b, z}\right)_{r} d z \\
\leq \frac{1}{2^{2 m} r^{2}} \sum_{i \neq j} p_{i} p_{j} \sum_{a \in A_{i}} \sum_{b \in A_{j}} \frac{8 r^{2}}{C_{\varepsilon, m} \varepsilon} \leq \frac{8}{C_{\varepsilon, m} \varepsilon}
\end{aligned}
$$

By combining 2.9 and 2.10 we have

$$
J(r) \leq \frac{8}{C_{\varepsilon, m} \varepsilon}+\beta \max _{i} J\left(\frac{r}{\lambda_{i, \min }(1-\varepsilon)}\right)
$$

where $\beta=\sum_{i=1}^{l} p_{i}^{2} \frac{(1+\varepsilon) \lambda_{i, \max }}{\left((1-\varepsilon) \lambda_{i, \min }\right)^{2}}$ is less than 1 by 1.6 .

We define recursively a strictly decreasing sequence $r_{k}$. Let $r_{0}<1 / 2$ be fixed. Assume that $r_{k-1}$ has been defined. Then we define $r_{k}=$ $(1-\varepsilon) \lambda_{i_{k}, \min } r_{k-1}$, where $i_{k}$ is chosen such that

$$
\max _{i} J\left(\frac{r_{k}}{(1-\varepsilon) \lambda_{i, \min }}\right)=J\left(\frac{r_{k}}{(1-\varepsilon) \lambda_{i_{k}, \min }}\right)=J\left(r_{k-1}\right) .
$$

Hence $r_{k}=r_{0}(1-\varepsilon)^{k} \prod_{n=1}^{k}\left(\lambda_{i_{n}, \min }\right)$.

We note that $r_{k}$ is a well defined sequence. By induction and (2.11), we have

$$
J\left(r_{k}\right) \leq \frac{8}{C_{\varepsilon, m} \varepsilon} \frac{1-\beta^{k}}{1-\beta}+\beta^{k} J\left(r_{0}\right)
$$

for every $k \geq 1$. Hence by (2.1), 2.2$)$ and $(2.12)$ we get

$$
\begin{aligned}
\left\|\nu_{\varepsilon, m}\right\|_{2}^{2} & \leq 4 \liminf _{r \rightarrow 0} J(r) \leq 4 \liminf _{k \rightarrow \infty} J\left(r_{k}\right) \\
& \leq \frac{32}{C_{\varepsilon, m} \varepsilon} \frac{1}{1-\sum_{i=1}^{l} p_{i}^{2} \frac{(1+\varepsilon) \lambda_{i, \max }}{\left((1-\varepsilon) \lambda_{i, \min }\right)^{2}}} .
\end{aligned}
$$

We now use the fact that a closed ball in the Hilbert space $L^{2}$ is compact in the weak topology. (See for instance Theorem V.2.1 in Yosida's book [16.) Hence, if $h_{\nu_{\varepsilon, m}}$ is the density of $\nu_{\varepsilon, m}$, then $h_{\nu_{\varepsilon}, m}$ is in $L^{2}$, and from the above we know that there is a constant $C_{\varepsilon}^{\prime}$ such that $\left\|h_{\nu_{\varepsilon, m}}\right\|_{2} \leq C_{\varepsilon}^{\prime} / \sqrt{\varepsilon}$. 
By the compactness statement above, there is an $h$ with $\|h\|_{2} \leq C_{\varepsilon}^{\prime} / \sqrt{\varepsilon}$ such that some subsequence of $h_{\nu_{\varepsilon, m}}$ converges weakly to $h$. Moreover $h$ defines a probability measure since $1=\int 1 \cdot h_{\nu_{\varepsilon, m}} d \mathcal{L}_{3} \rightarrow \int 1 \cdot h d \mathcal{L}_{3}$.

Since $\nu_{\varepsilon, m}$ converges weakly to $\nu_{\varepsilon}$ it follows that $\nu_{\varepsilon}$ has density in $L^{2}$ and

$$
\left\|\nu_{\varepsilon}\right\|_{2} \leq \frac{1}{\sqrt{\varepsilon}} C_{\varepsilon}^{\prime}
$$

where

$$
\begin{aligned}
C_{\varepsilon}^{\prime} & =\sqrt{\frac{32}{\left(1-\sum_{i=1}^{l} p_{i}^{2} \frac{(1+\varepsilon) \lambda_{i, \max }}{\left((1-\varepsilon) \lambda_{i, \min }\right)^{2}} C_{\varepsilon}^{\prime \prime}\right.}} \\
C_{\varepsilon}^{\prime \prime} & =\lim _{m \rightarrow \infty} C_{\varepsilon, m}=\min _{i \neq j}\left\{\frac{\left|a_{i}-a_{j}\right|+\varepsilon\left(-\left|a_{i}+a_{j}\right|-2\right)}{1-\varepsilon^{2}}\right\} .
\end{aligned}
$$

3. Proof of Theorem 1.5. We do not give the whole proof of Theorem 1.5, because it is similar to the proof of Theorem 1.2 . We only prove a modification of Lemma 2.2, which is important as it proves transversality.

First we define a new dynamical system $\widetilde{g}_{\varepsilon, m}: Q \rightarrow Q$, similar to the dynamical system $g_{\varepsilon, m}: Q \rightarrow Q$. Let $Q_{i, k}$ and $A_{i, k}$ be as in Section 2, Let $\widetilde{g}_{\varepsilon, m}: Q \rightarrow Q$ be defined by

$$
\widetilde{g}_{\varepsilon, m}:(x, y, z) \mapsto\left(\widetilde{d}(z) x+a_{i}(1-\widetilde{d}(z)), \frac{1}{p_{i}} y+b(y), 2^{m} z+c(z)\right)
$$

for $(x, y, z) \in Q_{i}$, where

$$
\begin{array}{ll}
\widetilde{d}(z)=\lambda_{i}+2^{m} \varepsilon\left(z-\left(-1+(k+1 / 2) 2^{-m+1}\right)\right) & \text { for }(x, y, z) \in Q_{i, k}, \\
b(y)=1-\frac{1}{p_{i}}\left(-1+2 \sum_{j=1}^{i} p_{j}\right) & \text { for }(x, y, z) \in Q_{i, k}, \\
c(z)=2^{m}-2 k-1 & \text { for }(x, y, z) \in Q_{i, k} .
\end{array}
$$

Hence the only difference between $\widetilde{g}_{\varepsilon, m}$ and $g_{\varepsilon, m}$ is in the first coordinate, where the perturbation of $f_{i}$ is made. Figure 1 also serves to visualise the action of $\widetilde{g}_{\varepsilon, m}$.

We define the cones

$$
C_{p}=\left\{(u, v, w) \in T_{p} Q:\left|\frac{u}{w}\right|,\left|\frac{v}{w}\right|<\frac{2^{m+1} \varepsilon}{2^{m}-\lambda_{\max }-\varepsilon}\right\},
$$

where $p \in Q$ and $\lambda_{\max }=\max _{i} \lambda_{i}$. Similar to Lemma2.2, we show that these cones define a family of unstable cones, and that a certain transversality property holds.

Lemma 3.1. Suppose that (1.9) holds. The cones $C_{p}$ form a family of unstable cones, that is, $d_{p} \widetilde{g}_{\varepsilon, m}\left(C_{p}\right) \subset C_{\widetilde{g}_{\varepsilon, m}(p)}$. 
Moreover, for sufficiently large $m$ and every sufficiently small $\varepsilon>0$, if $\zeta_{1} \subset Q_{\xi_{1}}$ and $\zeta_{2} \subset Q_{\xi_{2}}$ are two line segments with tangents in $C_{p}$ such that $\xi_{1} \in A_{i}$ and $\xi_{2} \in A_{j}, i \neq j$, then if $\widetilde{g}_{\varepsilon, m}\left(\zeta_{1}\right)$ and $\widetilde{g}_{\varepsilon, m}\left(\zeta_{2}\right)$ intersect, and if $\left(u_{1}, v_{1}, 1\right)$ and $\left(u_{2}, v_{2}, 1\right)$ are tangents to $\widetilde{g}_{\varepsilon, m}\left(\zeta_{1}\right)$ and $\widetilde{g}_{\varepsilon, m}\left(\zeta_{2}\right)$ respectively, there exists a constant $C_{\varepsilon, m}$, depending on $\varepsilon$ and $m$, but bounded away from 0 and infinity, such that $\left|u_{1}-u_{2}\right|>C_{\varepsilon, m} \varepsilon$.

Proof. The Jacobian of $\widetilde{g}_{\varepsilon, m}$ is

$$
d_{p} \widetilde{g}_{\varepsilon, m}=\left(\begin{array}{ccc}
\widetilde{d}(z) & 0 & 2^{m} \varepsilon\left(x-a_{i}\right) \\
0 & 1 / p_{i} & 0 \\
0 & 0 & 2^{m}
\end{array}\right),
$$

where $p=(x, y, z) \in Q_{i, k}$. If $(u, v, w) \in C_{p}$, then

$$
d_{p} \widetilde{g}_{\varepsilon, m}(u, v, w)=\left(\begin{array}{c}
\widetilde{d}(z) u+2^{m} \varepsilon\left(x-a_{i}\right) w \\
\left(1 / p_{i}\right) v \\
2^{m} w
\end{array}\right) .
$$

The estimate

$$
\begin{aligned}
\frac{\left|\widetilde{d}(z) u+2^{m} \varepsilon\left(x-a_{i}\right) w\right|}{\left|2^{m} w\right|} & \leq \frac{\widetilde{d}(z)|u|}{2^{m}|w|}+2 \varepsilon \\
& \leq \frac{\lambda_{i}+\varepsilon}{2^{m}} \frac{2^{m+1} \varepsilon}{2^{m}-\lambda_{\max }-\varepsilon}+2 \varepsilon \leq \frac{2^{m+1} \varepsilon}{2^{m}-\lambda_{\max }-\varepsilon}
\end{aligned}
$$

shows that $d_{p} \widetilde{g}_{\varepsilon, m}\left(C_{p}\right) \subset C_{\widetilde{g}_{\varepsilon, m}(p)}$. Now we prove the other statement of the lemma. Assume that $p=\left(x_{p}, y_{p}, z_{p}\right) \in Q_{i}$ and $q=\left(x_{q}, y_{q}, z_{q}\right) \in Q_{j}, i \neq j$, are such that $\widetilde{g}_{\varepsilon, m}(p)=\widetilde{g}_{\varepsilon, m}(q)=(x, y, z)$. Then

$$
p \in Q_{i} \Rightarrow d_{p} \widetilde{g}_{\varepsilon, m}:(u, v, 1) \mapsto 2^{m}\left(\frac{\widetilde{d}\left(z_{p}\right)}{2^{m}} u+\left(x_{p}-a_{i}\right) \varepsilon, \frac{v}{p_{i}}, 1\right),
$$

and

$$
x_{p}=\frac{x-a_{i}\left(1-\widetilde{d}\left(z_{p}\right)\right)}{\widetilde{d}\left(z_{p}\right)}, \quad x_{q}=\frac{x-a_{j}\left(1-\widetilde{d}\left(z_{q}\right)\right)}{\widetilde{d}\left(z_{q}\right)} .
$$

Let $\widetilde{\Delta}_{i}=\frac{2\left(\lambda_{i}+\varepsilon\right)}{2^{m}-\lambda_{\max }-\varepsilon}$. Then

$$
d_{p} \widetilde{g}_{\varepsilon, m}\left(C_{p}\right) \subset\left\{w(u, v, 1): \frac{x-a_{i}}{\widetilde{d}\left(z_{p}\right)} \varepsilon-\widetilde{\Delta}_{i} \varepsilon \leq u \leq \frac{x-a_{i}}{\widetilde{d}\left(z_{p}\right)} \varepsilon+\widetilde{\Delta}_{i} \varepsilon\right\} .
$$

Therefore

$$
\left|u_{2}-u_{1}\right| \geq\left(\left|\frac{x-a_{i}}{\widetilde{d}\left(z_{p}\right)}-\frac{x-a_{j}}{\widetilde{d}\left(z_{q}\right)}\right|-\left(\widetilde{\Delta}_{i}+\widetilde{\Delta}_{j}\right)\right) \varepsilon
$$


The term

can be estimated by

$$
\left|\frac{x-a_{i}}{\widetilde{d}\left(z_{p}\right)}-\frac{x-a_{j}}{\widetilde{d}\left(z_{q}\right)}\right|
$$

$$
\left|\frac{x-a_{i}}{\widetilde{d}\left(z_{p}\right)}-\frac{x-a_{j}}{\widetilde{d}\left(z_{q}\right)}\right| \geq\left|\frac{\left|\widetilde{d}\left(z_{p}\right)-\widetilde{d}\left(z_{q}\right)\right||x|-\left|a_{j} \widetilde{d}\left(z_{p}\right)-a_{i} \widetilde{d}\left(z_{q}\right)\right|}{\widetilde{d}\left(z_{p}\right) \widetilde{d}\left(z_{q}\right)}\right| .
$$

Hence, this term is positive provided that

$$
\left|a_{j} \widetilde{d}\left(z_{p}\right)-a_{i} \widetilde{d}\left(z_{q}\right)\right|>\left|\widetilde{d}\left(z_{p}\right)-\widetilde{d}\left(z_{q}\right)\right| .
$$

Since $\lambda_{i}-\varepsilon \leq \widetilde{d}\left(z_{p}\right) \leq \lambda_{i}+\varepsilon$ and $\lambda_{j}-\varepsilon \leq \widetilde{d}\left(z_{q}\right) \leq \lambda_{j}+\varepsilon$, this is implied by 1.9 if $\varepsilon$ is sufficiently small.

If we let

$$
C_{\varepsilon, m}=\frac{1}{2} \min _{i \neq j} \frac{\left|a_{i} \lambda_{j}-a_{j} \lambda_{i}\right|-\left|\lambda_{i}-\lambda_{j}\right|}{\lambda_{i} \lambda_{j}}
$$

then

$$
\left|u_{2}-u_{1}\right| \geq C_{\varepsilon, m} \varepsilon
$$

provided that $\varepsilon$ is small and $m$ large.

In fact we can let

$$
C_{\varepsilon, m}=\sigma \min _{i \neq j} \frac{\left|a_{i} \lambda_{j}-a_{j} \lambda_{i}\right|-\left|\lambda_{i}-\lambda_{j}\right|}{\lambda_{i} \lambda_{j}}
$$

for some $0<\sigma<1$.

Acknowledgments. Research of Bárány was supported by the EU FP6 Research Training Network CODY.

\section{References}

[1] B. Bárány, M. Pollicott and K. Simon, Stationary measures for projective transformations: the Blackwell and Furstenberg measures, preprint, 2009.

[2] P. Diaconis and D. A. Freedman, Iterated random functions, SIAM Rev. 41 (1999), 45-76.

[3] A. H. Fan, K. Simon and H. Tóth, Contracting on average random IFS with repelling fixed point, J. Statist. Phys. 122 (2006), 169-193.

[4] K.-S. Lau, S.-M. Ngai and H. Rao, Iterated function systems with overlaps and self-similar measures, J. London Math. Soc. (2) 63 (2001), 99-116.

[5] J. Neunhäuserer, Properties of some overlapping self-similar and some self-affine measures, Acta Math. Hungar. 92 (2001), 143-161.

[6] S.-M. Ngai and Y. Wang, Self-similar measures associated with IFS with nonuniform contraction ratios, Asian J. Math. 9 (2005), 227-244.

[7] M. Nicol, N. Sidorov and D. Broomhead, On the fine structure of stationary measures in systems which contract on average, J. Theoret. Probab. 15 (2002), 715-730.

[8] Y. Peres and W. Schlag, Smoothness of projections, Bernoulli convolutions, and the dimension of exceptions, Duke Math. J. 102 (2000), 193-251. 
[9] Y. Peres, K. Simon and B. Solomyak, Absolute continuity for random iterated function systems with overlaps, J. London Math. Soc. (2) 74 (2006), 739-756.

[10] Y. Peres and B. Solomyak, Self-similar measures and intersections of Cantor sets, Trans. Amer. Math. Soc. 350 (1998), 4065-4087.

[11] T. Persson, On random Bernoulli convolutions, Dynam. Systems, to appear.

[12] Ya. Pesin, Dynamical systems with generalized hyperbolic attractors: hyperbolic, ergodic and topological properties, Ergodic Theory Dynam. Systems 12 (1992), 123151.

[13] J. Schmeling and S. Troubetzkoy, Dimension and invertibility of hyperbolic endomorphisms with singularities, Ergodic Theory Dynam. Systems 18 (1998), 12571282.

[14] K. Simon, B. Solomyak and M. Urbański, Invariant measures for parabolic IFS with overlaps and random continued fractions, Trans. Amer. Math. Soc. 353 (2001), $5145-5164$.

[15] M. Tsujii, Fat solenoidal attractor, Nonlinearity 14 (2001), 1011-1027.

[16] K. Yosida, Functional Analysis, Springer, Berlin, 1980.

Balázs Bárány

Department of Stochastics

Institute of Mathematics

Technical University of Budapest

P.O. Box 91

1521 Budapest, Hungary

E-mail: balubsheep@gmail.com
Tomas Persson

Institute of Mathematics

Polish Academy of Sciences

Śniadeckich 8

P.O. Box 21

00-956 Warszawa, Poland E-mail: tomasp@impan.pl

Received 24 February 2009;

in revised form 28 June 2010 